den Ergebnissen der einzelnen Wiederholungen (12) errechnet wurden. In den Erläuterungen zu den beiden Figuren sind fur je eine Temperatur neben den absoluten Mittelwerten auch die mittleren Fehler (m) angegeben worden.

Das Optimum für die Höhe des Sproßsystems und des Trockengewichtes liegt bei Setaria pumila bei $26^{\circ}$. Bei dieser Temperatur vermag Senecio vulgaris unter den Versuchsbedingungen nicht auf die Dauer zu gedeihen. Bei dieser Art befindet sich das Optimum für die Höhe des Sproßsystems bei $7^{\circ}$, einer Temperatur, in der Setaria pumila nach ziemlich kurzer Zeit abstarb. Hiervon weichen die optimalen Temperaturen von bestimmten, für die Fertilität kennzeichnenden Merkmalen ab. Diese liegen bei Setaria pumila bei $20^{\circ}$ (Anzahl der Blütenstände) und $23^{\circ}$ (Anzahl der Ährchen pro Blütenstand), bei Senecio vulgaris bei $10^{\circ}$ (Zahl der fertilen Früchte pro Körbchen). Es ergibt sich somit bei beiden Arten eine deutliche Verschiebung in den Bereich mittlerer Temperaturen gegeniiber dem Optimum für Merkmale der vegetativen Entwicklung. Die Erscheinung, daß die optimalen Temperaturen für Eigenschaften der vegetativen Entwicklung in extremeren Temperaturbereichen liegen als diejenigen der Fertilität, wurde auch noch bei weiteren Arten festgestellt.

Earhart Plant Research Laboratory, Division of Biology, California Institute of Technology, Pasadena, California

Eingegangen and 15. Oktober 1957 RÜDIGER KNAPP

1) WENT, F.W.: Chron, bot. 12, 89 (1950). - 2) KNAPP, R. Ber. dtsch. bot. Ges. 69, 399 (1956).

\section{Zur Frage der höchst zulässigen Strahlendosis}

Die Ermittelung der höchst zulässigen Strahlendosis ist an biologische Versuche gebunden. Als Testmaterial wählten wir Gewebekulturen von menschlichen Tumorzellen (Stamm HeLa). Hinsichtlich der Züchtungstechnik sei auf eine frühere

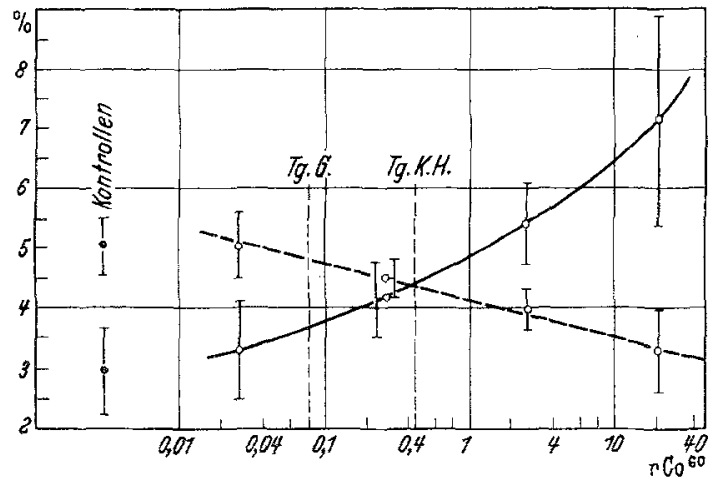

Fig. 1. Einwirkung von $\mathrm{Co}^{80}$ auf Gewebekulturen von HeLaZellen. - Schädigungsquotient, ...... Mitosehäufigkeit. $T g . G .=$ Toleranzgrenze (Ganzkörperbestrahlung). Tg.K.H. = Toleranzgrenze (Kopf- und Handbestrablung)

Veröffentlichung verwiesen ${ }^{1}$ ). Als Strahlenquelle dienten Co ${ }^{60}$-Perlen, deren Anzahl und deren Abstand von den Kulturen in Rollertubes so variert wurde, daß vier verschieden starke Dosen im Bereich von $10 \mathrm{r}$ bis $0,01 \mathrm{r} / \mathrm{Tag}$ zur Einwirkung kamen. Die Kulturen wurden der Dauerbestrahlung iber 48 Std ausgesetzt. Pro Versuch wurden je 8 Kulturen verwendet und von jeder Kultur 1000 Zellen ausgezählt, so daß jeder Wert auf einem Mittel von 8000 Zellen basiert. Ausgewertet wurden die Mitosehänfigkeit und der Schädigungsquotient, errechnet aus der Anzahl der

(path. Mitosen + Cytolysen-Kernpyknosen +Nebenkerne) $\times 100$ Gesamtzahl der Mitosen

Die bei den verschiedenen Dosen erhaltenen Mittelwerte und deren Streuung sind in Fig. 1 aufgetragen. Im halblogarithmischen Maßstab ergibt die Mitosehäufigkeit mit steigender Dosis eine abfallende gerade Linie. Der Schädigungsquotient steigt mit zunehmender Dosis verstärkt an. Die Beurteilung der Unterschiede $z$ wischen den einzelnen $z \mathfrak{u}$ vergleichenden Dosen erfolgte nach der $t$-Verteilung nach FISHER für verschiedene Sicherungsgrenzen von $P$. Wie aus der Tabelle 1

Tabelle 1. Mitosehäufigkeit (I) und Schädigungsquotient (II) von Dosis zu Dosis

\begin{tabular}{|c|c|c|c|c|c|c|c|}
\hline & $M_{1}-M_{2}$ & $M_{D}$ & $\sigma_{D}$ & $P$ & $t$ & $\sigma_{D} \cdot t$ & $M_{D} \leq \sigma_{D} \cdot t$ \\
\hline \multirow{3}{*}{1} & $20 \mathrm{r}-2 \mathrm{r}$ & 0,69 & 0,26 & $2 \%$ & 2,6 & 0,67 & $>$ \\
\hline & $2 \mathrm{r}-0,2 \mathrm{r}$ & 0,51 & 0,15 & $1 \%$ & 3,0 & 0,45 & $>$ \\
\hline & $0,2 \mathrm{r}-0,02 \mathrm{r}$ & 0,55 & 0,22 & $5 \%$ & 2,1 & 0,46 & $>$ \\
\hline \multirow{3}{*}{ II } & $20 \mathrm{I}-2 \mathrm{I}$ & 1,7 & 0,66 & $5 \%$ & 2,1 & $\{, 38$ & $>$ \\
\hline & $2 \mathrm{r}-0,2 \mathrm{r}$ & 1,2 & 0,33 & $0,27 \%$ & 3,6 & 1,18 & $>$ \\
\hline & $0,2 \mathrm{r}-0,02 \mathrm{r}$ & 0,9 & 0,37 & $5 \%$ & 2,1 & 0,77 & $>$ \\
\hline
\end{tabular}

ersichtlich ist, lassen sich die Werte für die Mitosehäufigkeiten und die Schädigungsquotienten von Dosis zu Dosis gegeneinander statistisch sichern. Zwischen den Werten der Kontrollen und denjenigen nach einer Einstrahlung von $0,026 \mathrm{r}$ 48 Std besteht kein Unterschied.

In die Abbildung wurden die zur Zeit gültigen Grenzen für die Strahlentoleranz des Menschen (entsprechend den Versuchszeiten) eingetragen (Kopf und Hände: 1,5 I/Woche; Ganzkörperbestrahlung: 0,3 r/Woche). Aus der Lage dieser Werte läßt sich entnehmen, daß die Toleranzgrenzen in einem Dosisbereich liegen, für den gegenüber den unbestrahlten Kontrollen signifikante Schädigungen ermittelt wurden.

Bezugnehmend auf frïhere Untersuchungen ${ }^{1}$ \}äßt sich feststellen, daß eine einmalige Dosis von $250 \mathrm{r}(40 \mathrm{r} / \mathrm{min}$, $15 \mathrm{MeV}$ Elektronen) an HeLa Zellen nach 48 Std die gleiche Mitosehemmung aufweist wie nach einer kontinuierlichen Einstrahlung von $0,2 \mathrm{r}$ ! Auch der Schädigungsquotient liegt nach $250 \mathrm{r}$ nur 2,5mal höher als nach $0,2 \mathrm{r}$ !

Die Möglichkeit, mit einer kontinuierlichen Strahlenbelastung von $0,1 \mathrm{r} / \mathrm{Tag}$ eine signifikante Verminderung der Mitosehäufiglkeit und eine Steigerung der Gesamtschädigungen der Zellen gegenüber den unbestrahlten Kontrollen hervorzurufen, weist darauf hin, daß die nach den Empfehlungen der internationalen Kommission für radiologische Einheiten festgelegte zulässige Strahlenbelastung noch nicht endgültig sein kann und weiterer experimenteller Unterbauung bedarf.

Tübingen, Medizinisches Strahleninstitut der Universität G. Breitling und K. Peters

Eingegangen am 8. November 1957

1) Peters, K.: Fortschr. Röntgenstr, (im Druck).

\title{
Bespredhungen
}

Dehlinger, U.: Theoretische Metallkunde. (Reine und angewandte Metallkunde in Einzeldarstellungen, Bd. 13.) BerlinGöttingen-Heidelberg: Springer 1955. V, 250 S. u. $82 \mathrm{Abb}$. Gr.-80. Gzl. DM 27.-.

Die theoretische Deutung der Metall-Probleme auf physikalischer Grundlage hat in der letzten Zeit wesentliche Fortschritte gemacht. Eine moderne Darstellung dieses Gebietes in deutscher Sprache ist daher sehr zu begrüßen, zumal sie aus der Feder eines Mannes stammt, der nicht nur das theoretische Rüstzeug beherrscht, sondern auch mit dem ungeheuren Tatsachenmaterial vertraut ist.

Naturgemäß beginnt eine solche Darstellung mit einer eingehenden Elektronentheorie der Metalle und Legierungen. Sie nimmt etwa die erste Hälfte des vorliegenden Bandes in An- spruch. Die zweite Hälfte ist der Diskussion von physikalischen Eigenscharten, Umwandlungserscheinungen und Gitterbaufehlern gewidmet.

Die moderne Literatur ist weitgehend verarbeitet. Daß im allgemeinen die im eigenen Institut erarbeiteten Ergebnisse etwas mehr im Vordergrund stehen, ist nur natürlich. Der auf dem Gebiet der Metallphysik schon etwas Fortgeschrittene wird dieses Buch mit großem Nutzen zur Hand nehmen, denn der Verf. führt den Leser mit auBerordentlichem Geschick an einem originellen theoretischen Geländer durch den experimentellen Erfahrungsbereich. Für einen Anfänger sind die Ansprüche, die an den Leser gestellt werden, zu groß.

An manchen Stellen würde man sich eine eingehendere Darstellung wünschen, so bei den elektronentheoretischen 